\title{
MULTIFRACTAL ANALYSIS OF THE BIRKHOFF SUMS OF SAINT-PETERSBURG POTENTIAL
}

\author{
DONG HAN KIM, LINGMIN LIAO, MICHA€ RAMS, AND BAO-WEI WANG
}

\begin{abstract}
Let $((0,1], T)$ be the doubling map in the unit interval and $\varphi$ be the Saint-Petersburg potential, defined by $\varphi(x)=2^{n}$ if $x \in\left(2^{-n-1}, 2^{-n}\right.$ for all $n \geq 0$. We consider asymptotic properties of the Birkhoff sum $S_{n}(x)=$ $\varphi(x)+\cdots+\varphi\left(T^{n-1}(x)\right)$. With respect to the Lebesgue measure, the SaintPetersburg potential is not integrable and it is known that $\frac{1}{n \log n} S_{n}(x)$ converges to $\frac{1}{\log 2}$ in probability. We determine the Hausdorff dimension of the level set $\left\{x: \lim _{n \rightarrow \infty} S_{n}(x) / n=\alpha\right\} \quad(\alpha>0)$, as well as that of the set $\left\{x: \lim _{n \rightarrow \infty} S_{n}(x) / \Psi(n)=\alpha\right\}(\alpha>0)$, when $\Psi(n)=n \log n, n^{a}$ or $2^{n^{\gamma}}$ for $a>1, \gamma>0$. The fast increasing Birkhoff sum of the potential function $x \mapsto 1 / x$ is also studied.
\end{abstract}

\section{INTRODUCTION}

Let $T$ be the doubling map on the unit interval $(0,1]$ defined by

$$
T x=2 x-\lceil 2 x\rceil+1,
$$

where $\lceil x\rceil$ is the smallest integer larger than or equal to $x$. Let $\epsilon_{1}$ be the function defined by $\epsilon_{1}(x)=\lceil 2 x\rceil-1$ and $\epsilon_{n}(x):=\epsilon_{1}\left(T^{n-1} x\right)$ for $n \geq 2$. Then each real number $x \in(0,1]$ can be expanded into an infinite series as

$$
x=\frac{\epsilon_{1}(x)}{2}+\cdots+\frac{\epsilon_{n}(x)}{2^{n}}+\cdots .
$$

We call (1.1) the binary expansion of $x$ and also write it as

$$
x=\left[\epsilon_{1}(x) \epsilon_{2}(x) \ldots\right] .
$$

The Saint-Petersburg potential is a function $\varphi:(0,1] \rightarrow \mathbb{R}$ defined as

$$
\varphi(x)=2^{n} \text { if } x \in\left(2^{-n-1}, 2^{-n}\right], \forall n \geq 0 .
$$

We remark that the definition of $\varphi$ is equivalent to

$$
\varphi(x)=2^{n} \text { where } n \geq 0 \text { is the smallest integer such that } \epsilon_{n+1}(x)=1 .
$$

and is also equivalent to

$$
\varphi(x)=2^{n} \text { if the binary expansion of } x \text { begins with } 0^{n} 1,
$$

where $0^{n}(n \geq 0)$ means a block with $n$ consecutive zeros.

The name of Saint-Petersburg potential is motivated by the famous Saint-Petersburg game in probability theory. The Saint-Petersburg potential is of infinite expectation with respect to the Lebesgue measure. Furthermore, it increases exponentially fast near to the point 0 .

\footnotetext{
2010 Mathematics Subject Classification. Primary 37E05 Secondary 28A80, 37D20.

Key words and phrases. Saint-Petersburg potential, Hausdorff dimension, multifractal analysis.

D.K. was supported by the NRF of Korea (NRF-2015R1A2A2A01007090, NRF2017K1A3A1A21013650). M.R. was supported by National Science Centre grant 2014/13/B/ST1/01033 (Poland). B.W. was partially supported by NSFC N0. 11471030.
} 
In this paper, we are concerned with the following Birkhoff sums of the SaintPetersburg potential:

$$
\forall n \geq 1, \quad S_{n}(x):=\varphi(x)+\varphi(T(x))+\cdots+\varphi\left(T^{n-1}(x)\right), \quad x \in(0,1] .
$$

Let

$$
I=\left\{x \in(0,1]: \epsilon_{1}(x)=1\right\} .
$$

Define the hitting time of $x \in(0,1]$ to $I$ as

$$
n(x):=\inf \left\{n \geq 0: T^{n} x \in I\right\} .
$$

Then

$$
n(x)=n \quad \text { if } x \in\left(\frac{1}{2^{n+1}}, \frac{1}{2^{n}}\right], \text { for all } n \geq 0 .
$$

Using $n(x)$, we define a new dynamical system $\widehat{T}:(0,1] \rightarrow(0,1]$ by

$$
\widehat{T}(x)=T^{n(x)+1}(x)=2^{n+1}\left(x-\frac{1}{2^{n+1}}\right) \text { if } x \in\left(\frac{1}{2^{n+1}}, \frac{1}{2^{n}}\right] \text {, for all } n \geq 0,
$$

called the acceleration of $T$, in order that $\varphi$ and $\varphi \circ \widehat{T}$ are independent. Let

$$
\widehat{S}_{n}(x):=\varphi(x)+\varphi(\widehat{T}(x))+\cdots+\varphi\left(\widehat{T}^{n-1}(x)\right), \quad x \in(0,1] .
$$

The convergence in probability of $\widehat{S}_{n}(x)$ is well known (e.g. 6, p.253]) which states that for any $\epsilon>0$, the Lebesgue measure $\lambda$ of

$$
\left\{x \in(0,1]:\left|\frac{\widehat{S}_{n}(x)}{n \log n}-\frac{1}{\log 2}\right| \geq \epsilon\right\}
$$

tends to 0 as $n \rightarrow \infty$.

Let $\left\{\Psi_{n}\right\}_{n \geq 1}$ be an increasing sequence such that $\Psi_{n} \rightarrow \infty$ as $n \rightarrow \infty$. Then it was shown in [5] that Lebesgue almost surely either

$$
\lim _{n \rightarrow \infty} \frac{\widehat{S}_{n}(x)}{\Psi_{n}}=0 \text { or } \quad \limsup _{n \rightarrow \infty} \frac{\widehat{S}_{n}(x)}{\Psi_{n}}=\infty,
$$

according as

$$
\sum_{n \geq 1} \lambda\left(\left\{x \in(0,1]: \varphi(x) \geq \Psi_{n}\right\}\right)<\infty \quad \text { or } \quad=\infty
$$

Let $n_{1}=n_{1}(x)=n(x)+1$ and $n_{k}=n_{k}(x)=n_{1}\left(\widehat{T}^{k-1} x\right)=n\left(\widehat{T}^{k-1} x\right)$ for $k \geq 2$. It is direct to see that

$$
\forall \ell \geq 1, \quad S_{n_{1}+\cdots+n_{\ell}}(x)=2 \widehat{S}_{\ell}(x)-\ell .
$$

Moreover, the ergodicity of $T$ ( of $\widehat{T}$ ) implies Lebesgue almost surely

$$
\lim _{\ell \rightarrow \infty} \frac{n_{1}(x)+\cdots+n_{\ell}(x)}{\ell}=\int_{0}^{1}(n(x)+1) d \lambda(x)=2 .
$$

Combining these two facts together, we obtain the same convergence results as above if we replace $\widehat{S}_{n}$ by $S_{n}$. In particular, the average $S_{n}(x) /(n \log n)$ converges to $1 / \log 2$ in probability, and almost surely (with respect to the Lebesgue measure) either

according as

$$
\lim _{n \rightarrow \infty} \frac{S_{n}(x)}{\Psi_{n}}=0 \quad \text { or } \quad \limsup _{n \rightarrow \infty} \frac{S_{n}(x)}{\Psi_{n}}=\infty,
$$

$$
\sum_{n \geq 1} \lambda\left(\left\{x \in(0,1]: \varphi(x) \geq \Psi_{n}\right\}\right)<\infty \quad \text { or } \quad=\infty
$$


where $\left\{\Psi_{n}\right\}_{n \geq 1}$ is an increasing sequence such that $\Psi_{n} \rightarrow \infty$ as $n \rightarrow \infty$. Recall that $\varphi$ has infinite expectation with respect to the Lebesgue measure. We thus have $S_{n}(x) / n$ converges to infinity for Lebesgue almost all points.

In this article, we want to further study the asymptotic behavior of of the Birkhoff sum $S_{n}(x)$ of the Saint-Petersburg potential. We give a complete multifractal analysis of $S_{n}(x)$.

First, for any $\alpha \geq 1$, we consider the level set

$$
E(\alpha)=\left\{x \in(0,1]: \lim _{n \rightarrow \infty} \frac{1}{n} S_{n}(x)=\alpha\right\} .
$$

For $t \in \mathbb{R}$ and $q>0$, define

$$
P(t, q):=\log \sum_{j=1}^{\infty} 2^{-t j-q\left(2^{j}-1\right)} .
$$

Then $P$ is a real-analytic function. Furthermore, for each $q>0$, there is a unique $t(q)>0$ such that $P(t(q), q)=0$. This function $q \mapsto t(q)$ is real-analytic, strictly decreasing and convex.

Denote by $\operatorname{dim}_{H}$ the Hausdorff dimension. The function $\alpha \mapsto \operatorname{dim}_{H} E(\alpha)$, called the Birkhoff spectrum of the Saint-Petersburg potential $\varphi$, is proved to be the Legendre transformation of the function $q \mapsto t(q)$.

Theorem 1.1. For any $\alpha \geq 1$ we have

$$
\operatorname{dim}_{H} E(\alpha)=\inf _{q>0}\{t(q)+q \alpha\} .
$$

Consequently, $\operatorname{dim}_{H} E(1)=0$ and the function $\alpha \mapsto \operatorname{dim}_{H} E(\alpha)$ is real-analytic, strictly increasing, concave, and has limit 1 as $\alpha \rightarrow \infty$.

The Birkhoff spectrum of a continuous potential was obtained for full shifts [14, for topologically mixing subshifts of finite type [4, and for repellers of a topologically mixing $C^{1+\epsilon}$ expanding map 2. A continuous potential in a compact space is bounded, hence these classical results are all for bounded potentials. Our Theorem 1.1 gives a Birkhoff spectrum for an unbounded function with a singular point. To prove Theorem 1.1 we will transfer our question to a Birkhoff spectrum problem of an interval map with infinitely many branches and we will apply the techniques developed in [9] for continued fraction dynamical system and in [8] for general expanding interval maps with infinitely many branches.

We also study the Birkhoff sums $S_{n}(x)$ of fast increasing rates. Let $\Psi: \mathbb{N} \rightarrow \mathbb{N}$ be an increasing function. For $\beta \in[0, \infty]$, consider the level set

$$
E_{\Psi}(\beta):=\left\{x \in(0,1]: \lim _{n \rightarrow \infty} \frac{1}{\Psi(n)} S_{n}(x)=\beta\right\} .
$$

Theorem 1.2. If $\Psi(n)$ is one of the following

$$
\Psi(n)=n \log n, \Psi(n)=n^{a}(a>1), \Psi(n)=2^{n^{\gamma}}(0<\gamma<1 / 2),
$$

then for any $\beta \in[0, \infty], \operatorname{dim}_{H} E_{\Psi}(\beta)=1$.

If $\Psi(n)=2^{n^{\gamma}}$ with $1 / 2 \leq \gamma<1$, then for any $\beta \in(0, \infty)$, the set $E_{\Psi}(\beta)$ is empty, and $\operatorname{dim}_{H} E_{\Psi}(\beta)=1$ for $\beta=0,+\infty$.

If $\Psi(n)=2^{n^{\gamma}}$ with $\gamma \geq 1$, then for any $\beta \in(0, \infty]$, the set $E_{\Psi}(\beta)$ is empty, and $\operatorname{dim}_{H} E_{\Psi}(\beta)=1$ for $\beta=0$.

We remark that by the above discussion on the convergence of $S_{n}(x)$, for all cases in Theorem 1.2 the sets $E_{\Psi}(0)$ has full measure, and thus obviously has full Hausdorff dimension. 
From the definition of $S_{n}(x)$, we see that for the integer $n$ such that $\epsilon_{n}(x)=1$, one has $S_{n}(x)=S_{n-1}(x)+1$, which implies for all $x, \liminf _{n \rightarrow \infty} \frac{S_{n}(x)}{S_{n-1}(x)}=1$. Thus if $\liminf _{n \rightarrow \infty} \frac{\Psi(n)}{\Psi(n-1)}>1$, then for any $\beta \in(0, \infty)$, the set $E_{\Psi}(\beta)$ is empty. By the definition of $S_{n}(x)$, we can also check that for all $x$, for the integer $n$ such that $\epsilon_{n}(x)=1$, we have $S_{n}(x) \leq 2^{n}-1$. This implies $\liminf _{n \rightarrow \infty} S_{n}(x) / 2^{n} \leq 1$ (See also the formula (3.15) in Section 3). Hence, for 'regular' growth functions $\Psi$ we only need to consider exponential and subexponential growth rates.

However, if we pick a point $x$ with dyadic expansion consisting mostly of 0 's, with infinitely many 1's but in large distances from each other, then the Birkhoff sum $S_{n_{i}}(x)$ may grow arbitrarily fast on some subsequence $n_{i}$. Thus for any increasing $\Psi(n)$ there exists a point $x$ such that $\limsup _{n \rightarrow \infty} S_{n}(x) / \Psi(n)=\infty$.

Our study on the Saint-Petersburg potential is an attempt of multifractal analysis of unbounded potential functions on the doubling map dynamical system. However, the Saint-Petersburg potential is locally constant and not continuous. One might think of an another unbounded potential function $g: x \mapsto 1 / x$ which is close to the Saint-Petersburg potential but is continuous. In fact, our method for studying the fast increasing Birkhoff sum of Saint-Petersburg potential also works for the fast increasing Birkhoff sum of the potential $g: x \mapsto 1 / x$.

Denote by $S_{n} g(x)$ the Birkhoff sum

$$
S_{n} g(x):=g(x)+g(T(x))+\cdots+g\left(T^{n-1}(x)\right), \quad x \in(0,1] .
$$

For $\beta \in[0, \infty]$, let

$$
F_{\Psi}(\beta):=\left\{x \in(0,1]: \lim _{n \rightarrow \infty} \frac{1}{\Psi(n)} S_{n} g(x)=\beta\right\} .
$$

Theorem 1.3. If $\Psi(n)$ is one of the following

$$
\Psi(n)=n \log n, \Psi(n)=n^{a}(a>1), \Psi(n)=2^{n^{\gamma}}(0<\gamma<1 / 2),
$$

then for any $\beta \in[0, \infty], \operatorname{dim}_{H} F_{\Psi}(\beta)=1$.

If $\Psi(n)=2^{n^{\gamma}}$ with $1 / 2 \leq \gamma<1$, then for any $\beta \in(0, \infty)$, the set $F_{\Psi}(\beta)$ is empty, and $\operatorname{dim}_{H} F_{\Psi}(\beta)=1$ for $\beta=0,+\infty$.

If $\Psi(n)=2^{n^{\gamma}}$ with $\gamma \geq 1$, then for any $\beta \in(0, \infty]$, the set $F_{\Psi}(\beta)$ is empty, and $\operatorname{dim}_{H} F_{\Psi}(\beta)=1$ for $\beta=0$.

We remark that these multifractal analysis on the Birkhoff sums of fast increasing rates have been done for some special potentials in continued fraction dynamical system $([9,11,12])$.

\section{Birkhoff spectrum of the SAint-Petersburg Potential}

In this section, we will obtain the Birkhoff spectrum of the Saint-Petersburg potential, i.e. the Hausdorff dimension of the following level set:

$$
E(\alpha):=\left\{x \in(0,1]: \lim _{n \rightarrow \infty} \frac{1}{n} \sum_{j=0}^{n-1} \varphi\left(T^{j} x\right)=\alpha\right\} \quad(\alpha \geq 1) .
$$

We will transfer our question to a Birkhoff spectrum problem for an interval map with infinitely many branches. 
2.1. Transference lemma. Recall that the Saint-Petersburg potential $\varphi$ is given by

$$
\varphi(x)=2^{n}, \text { if } x=\left[0^{n} 1, \cdots\right]
$$

where $x=\left[\epsilon_{1} \epsilon_{2}, \cdots\right]$ denotes the digit sequence in the binary expansion of $x$. Recall also the definition of hitting time $n(x)$ and the acceleration $\widehat{T}$ of the doubling map $T$ in Section 1, Define a new potential function

$$
\phi(x):=2^{n(x)+1}-1, x \in(0,1] .
$$

In fact, $\phi$ is nothing but the function satisfying

$$
\phi(x)=\sum_{j=0}^{n(x)} \varphi\left(T^{j} x\right)
$$

With the notation $n_{1}=n(x)+1 \geq 1$, and $n_{k}=n\left(\widehat{T}^{k-1} x\right)+1$ for $k \geq 2$ given in Section 1, we have

$$
\phi(\widehat{T} x)=\sum_{j=0}^{n(\widehat{T} x)} \varphi\left(T^{j}(\widehat{T} x)\right)=\sum_{j=n_{1}}^{n_{2}-1} \varphi\left(T^{j} x\right) .
$$

Hence,

$$
\sum_{j=0}^{n_{1}+\cdots+n_{\ell}-1} \varphi\left(T^{j} x\right)=\sum_{k=0}^{\ell-1} \phi\left(\widehat{T}^{k} x\right)=2^{n_{1}}+\cdots+2^{n_{\ell}}-\ell
$$

Note that the derivative of $\widehat{T}$ satisfies

$$
\left|\widehat{T}^{\prime}\right|(x)=2^{n(x)+1}=2^{n_{1}}=\phi(x)+1 .
$$

We have

$$
n_{1}+\cdots+n_{\ell}=\sum_{k=0}^{\ell-1} \log _{2}\left|\widehat{T}^{\prime}\right|\left(\widehat{T}^{k} x\right)
$$

Recall the set in question:

$$
E(\alpha)=\left\{x \in(0,1]: \lim _{n \rightarrow \infty} \frac{1}{n} \sum_{j=0}^{n-1} \varphi\left(T^{j} x\right)=\alpha\right\} \quad(\alpha \geq 1) .
$$

Define

$$
\widetilde{E}(\alpha):=\left\{x \in(0,1]: \lim _{\ell \rightarrow \infty} \frac{\sum_{k=0}^{\ell-1} \phi\left(\widehat{T}^{k} x\right)}{\sum_{k=0}^{\ell-1} \log _{2}\left|\widehat{T}^{\prime}\right|\left(\widehat{T}^{k} x\right)}=\alpha\right\} \quad(\alpha \geq 1) .
$$

The following lemma shows the two level sets are the same.

Lemma 2.1. For all $\alpha \geq 1$, we have $E(\alpha)=\widetilde{E}(\alpha)$.

Proof. It is evident that $E(\alpha) \subset \widetilde{E}(\alpha)$, because, as discussed above,

$$
\frac{\sum_{k=0}^{\ell-1} \phi\left(\widehat{T}^{k} x\right)}{\sum_{k=0}^{\ell-1} \log _{2}\left|\widehat{T}^{\prime}\right|\left(\widehat{T}^{k} x\right)}=\frac{1}{n_{1}+\cdots+n_{\ell}} \sum_{j=0}^{n_{1}+\cdots+n_{\ell}-1} \varphi\left(T^{j} x\right) .
$$

Now, we show the other direction. Take an $x \in \widetilde{E}(\alpha)$, express $x$ in its binary expansion

$$
x=\left[0^{n_{1}-1} 10^{n_{2}-1} 1 \cdots 0^{n_{\ell}-1} 1 \cdots\right] .
$$

In fact, $n_{\ell}-1$ is the recurrence time for $n\left(\widehat{T}^{\ell-1} x\right)$, for each $\ell \geq 1$. 
By (2.1), we have, at present,

$$
\lim _{\ell \rightarrow \infty} \frac{1}{n_{1}+\cdots+n_{\ell}} \sum_{j=0}^{n_{1}+\cdots+n_{\ell}-1} \varphi\left(T^{j} x\right)=\alpha .
$$

So, we are required to check it holds for all $n$.

For any $\epsilon>0$, there exists $\ell_{0} \in \mathbb{N}$ such that, for any $\ell \geq \ell_{0}$,

$$
\alpha-\epsilon \leq \frac{2^{n_{1}}+\cdots+2^{n_{\ell}}-\ell}{n_{1}+\cdots+n_{\ell}} \leq \alpha+\epsilon .
$$

For any $n_{1}+\cdots+n_{\ell}<n<n_{1}+\cdots+n_{\ell}+n_{\ell+1}$ with $\ell \geq \ell_{0}$, it is trivial that

$$
\frac{2^{n_{1}}+\cdots+2^{n_{\ell}}-\ell}{n_{1}+\cdots+n_{\ell}+n_{\ell+1}} \leq \frac{1}{n} \sum_{j=0}^{n-1} \varphi\left(T^{j} x\right) \leq \frac{2^{n_{1}}+\cdots+2^{n_{\ell}}+2^{n_{\ell+1}}-\ell-1}{n_{1}+\cdots+n_{\ell}} .
$$

Thus, it suffices to show that

$$
2^{n_{\ell+1}}=o\left(n_{1}+\cdots+n_{\ell}\right)
$$

which also implies

$$
n_{\ell+1}=o\left(n_{1}+\cdots+n_{\ell}\right) .
$$

Let $M_{0}$ be a large integer such that, for all $M \geq M_{0}, 2^{M} \geq 4 \alpha M$. So, when $n_{\ell+1} \leq M_{0}$, there is nothing to prove. So, we always assume $2^{n_{\ell+1}} \geq 4 \alpha n_{\ell+1}$.

By (2.4), we have

$$
\begin{aligned}
2^{n_{1}}+\cdots+2^{n_{\ell}}-\ell & \geq(\alpha-\epsilon)\left(n_{1}+\cdots+n_{\ell}\right), \\
2^{n_{1}}+\cdots+2^{n_{\ell}}+2^{n_{\ell+1}}-\ell-1 & \leq(\alpha+\epsilon)\left(n_{1}+\cdots+n_{\ell}+n_{\ell+1}\right) .
\end{aligned}
$$

These give

$$
2^{n_{\ell+1}} \leq 2 \epsilon\left(n_{1}+\cdots+n_{\ell}\right)+(\alpha+\epsilon) n_{\ell+1}+1 .
$$

So, we have

$$
2^{n_{\ell+1}} \leq 4 \epsilon\left(n_{1}+\cdots+n_{\ell}\right)
$$

2.2. Dimension of $\widetilde{E}(\alpha)$. Now we calculate the Hausdorff dimension of the set $\widetilde{E}(\alpha)$. At first, we give a notation.

- For each finite word $w \in \bigcup_{n>1}\{0,1\}^{n}$ of length $n$, a $T$-dyadic cylinder of order $n$ is defined as

$$
I_{n}(w)=\left\{x \in(0,1]:\left(\epsilon_{1}(x), \cdots, \epsilon_{n}(x)\right)=w\right\} .
$$

- For $\left(n_{1}, \cdots, n_{\ell}\right) \in(\mathbb{N} \backslash\{0\})^{\ell}$, a $\widehat{T}$-dyadic cylinder of order $\ell$ is defined as

$$
D_{\ell}\left(n_{1}, \cdots, n_{\ell}\right)=\left\{x \in(0,1]: n_{k}(x)=n_{k}, 1 \leq k \leq \ell\right\} .
$$

Proof of Theorem 1.1. To calculate the Hausdorff dimension of $\widetilde{E}(\alpha)$, we construct a suitable measure supported on $\widetilde{E}(\alpha)$. The Gibbs measures derived from the Ruelle-Perron-Frobenius transfer operator are good candidates for such a measure.

In fact, by considering the inverse branches $U_{i}: x \mapsto \frac{x+1}{2^{i+1}}(i \geq 0)$ of $\widehat{T}$, we can code the dynamical system $([0,1], \widehat{T})$ by the conformal infinite iterated function system $\left(U_{i}\right)_{i \geq 0}$ which satisfies the open set condition ([10, Section 1]).

Consider the potential function with two parameters

$$
\psi_{t, q}:=-t \log \left|\widehat{T}^{\prime}\right|-(\log 2) \cdot q \phi \quad(t \in \mathbb{R}, q>0) .
$$

Then $\left(\psi_{t, q} \circ U_{i}\right)_{i \geq 0}$ is a family of strong Hölder family ([10, Page 30]). Hence, we can define a Ruelle operator

$$
\mathcal{L}_{t, q} f(x):=\sum_{y \in \widehat{T}^{-1} x} e^{\psi_{t, q}(y)} f(y),
$$


on the Banach space of continuous functions on the corresponding infinite symbolic space $([10$, Page 31$])$.

By the Ruelle-Perron-Frobenius transfer operator theory 10, Theorems 2.9 and 2.10 ], for any $q>0$ (to satisfy the condition 2.2 of [10]), we can find an eigenvalue $\lambda_{t, q}$ and an eigenfunction $h_{t, q}$ for $\mathcal{L}_{t, q}$ and an eigenfunction $\nu_{t, q}$ for the conjugate operator $\mathcal{L}_{t, q}^{*}$. Then the pressure function $P(t, q)=\log \lambda_{t, q}$ and the ergodic Gibbs measure $\mu_{t, q}$ is given by $h_{t, q} \cdot \nu_{t, q}$.

The pressure function can be computed by (see [10, Pages 31 and 48])

$$
P(t, q)=\lim _{\ell \rightarrow \infty} \frac{1}{\ell} \log \left(\sum_{\left(n_{1}, \cdots, n_{\ell}\right) \in(\mathbb{N} \backslash\{0\})^{\ell}} \exp \sup _{x \in D_{\ell}\left(n_{1}, \cdots, n_{\ell}\right)}\left(S_{\ell} \psi_{t, q}(x)\right)\right) .
$$

Note that for all $n \geq 1$,

$$
\left|\widehat{T}^{\prime}\right|(x)=2^{n}, \text { and } \phi(x)=2^{n}-1 \text { if } x \in\left(\frac{1}{2^{n}}, \frac{1}{2^{n-1}}\right]=D_{1}(n) .
$$

Then for $x \in D_{\ell}\left(n_{1}, \cdots, n_{\ell}\right)$,

$$
S_{\ell} \psi_{t, q}(x)=-(\log 2) \cdot t \cdot \sum_{j=1}^{\ell} n_{j}+(\log 2) \cdot q \cdot \sum_{j=1}^{\ell}\left(2^{n_{j}}-1\right) .
$$

Thus

$$
\begin{gathered}
\sum_{\left(n_{1}, \cdots, n_{\ell}\right) \in(\mathbb{N} \backslash\{0\})^{\ell}} \exp \sup _{x \in D_{\ell}\left(n_{1}, \cdots, n_{\ell}\right)}\left(S_{\ell} \psi_{t, q}(x)\right) \\
=\sum_{n_{1}=1}^{\infty} \cdots \sum_{n_{\ell}=1}^{\infty}\left(\prod_{j=1}^{\ell} 2^{-t n_{j}} \cdot \prod_{j=1}^{\ell} 2^{q\left(2^{n_{j}}-1\right)}\right)=\left(\sum_{j=1}^{\infty} 2^{-t j-q\left(2^{j}-1\right)}\right)^{\ell} .
\end{gathered}
$$

Hence

$$
P(t, q)=\log \sum_{j=1}^{\infty} 2^{-t j-q\left(2^{j}-1\right)} .
$$

Now we calculate the local dimension of the Gibbs measure $\mu_{t, q}$. Let $D_{\ell}(x)$ be the $\widehat{T}$-dyadic cylinder containing $x$ of order $\ell$. By the Gibbs property of $\mu_{t, q}$,

$$
\begin{aligned}
\frac{\log \mu_{t, q}\left(D_{\ell}(x)\right)}{\log \left|D_{\ell}(x)\right|} & =\frac{S_{\ell} \psi_{t, q}(x)-\ell P(t, q)}{-S_{\ell} \log \left|\widehat{T}^{\prime}\right|(x)} \\
& =\frac{-t S_{\ell} \log \left|\widehat{T}^{\prime}\right|(x)-(\log 2) \cdot q S_{\ell} \phi(x)-\ell P(t, q)}{-S_{\ell} \log \left|\widehat{T}^{\prime}\right|(x)} \\
& =t+q \frac{S_{\ell} \phi(x)}{S_{\ell} \log _{2}\left|\widehat{T}^{\prime}\right|(x)}+\frac{\ell P(t, q)}{S_{\ell} \log \left|\widehat{T}^{\prime}\right|(x)} .
\end{aligned}
$$

- UPPer BOUND. For each $q>0$, let $t(q)$ be the the number such that $P(t(q), q)=0$. (The existence of $t(q)$ comes from the facts that $P(t, q)$ is realanalytic and that for fixed $q>0, P(t, q)>0$ when $t \rightarrow-\infty$ and $P(t, q)<0$ when $t \rightarrow+\infty$.) Then for all $x \in \widetilde{E}(\alpha)$, we have

$$
\begin{aligned}
\liminf _{r \rightarrow 0} \frac{\log \mu_{t, q}(B(x, r))}{\log r} & \leq \liminf _{\ell \rightarrow \infty} \frac{\log \mu_{t, q}\left(B\left(x,\left|D_{\ell}(x)\right|\right)\right)}{\log \left|D_{\ell}(x)\right|} \\
& \leq \liminf _{\ell \rightarrow \infty} \frac{\log \mu_{t, q}\left(D_{\ell}(x)\right)}{\log \left|D_{\ell}(x)\right|}=t(q)+q \alpha
\end{aligned}
$$


where for the second inequality the trivial inclusion $D_{\ell}(x) \subset B\left(x,\left|D_{\ell}(x)\right|\right)$ is used. By Billingsley Lemma (see e.g. [3, Proposition 4.9.]), this gives an upper bound of the Hausdorff dimension of $\widetilde{E}(\alpha)$. Thus we have

$$
\operatorname{dim}_{H} \widetilde{E}(\alpha) \leq \inf _{q>0}\{t(q)+q \alpha\} .
$$

- Lower Bound. By the real-analyticity of $P(t, q)$ and the implicit function theorem, the function $q \mapsto t(q)$ is also real-analytic. Thus there exists $q_{0}$ such that the following infimum is attained

$$
\inf _{q>0}\{t(q)+q \alpha\} .
$$

Then we have

$$
t^{\prime}\left(q_{0}\right)+\alpha=0 .
$$

To prove the lower bound, we first show two claims.

Claim (A): The measure $\mu_{t\left(q_{0}\right), q_{0}}$ is supported on $E_{\alpha}$.

On the one hand, since $P(t(q), q)=0$,

$$
\frac{\partial P}{\partial t} t^{\prime}(q)+\frac{\partial P}{\partial q}=0
$$

On the other hand, by the ergodicity of the measure $\mu_{t, q}$, we have for $\mu_{t, q}$ almost all $x$,

$$
\lim _{\ell \rightarrow \infty} \frac{S_{\ell} \phi(x)}{S_{\ell} \log _{2}\left|\widehat{T}^{\prime}\right|(x)}=\frac{\int \phi d \mu_{t, q}}{\int \log \left|\widehat{T}^{\prime}\right| d \mu_{t, q}} \cdot \log 2 .
$$

By Ruelle-Perron-Frobenius transfer operator theory ([10], Proposition 6.5),

$$
\int(\log 2) \cdot \phi d \mu_{t, q}=-\frac{\partial P}{\partial q} \quad \text { and } \quad \int \log \left|\widehat{T}^{\prime}\right| d \mu_{t, q}=-\frac{\partial P}{\partial t} .
$$

Thus by (2.8) and then (2.7), for $\mu_{t\left(q_{0}\right), q_{0}}$ almost all $x$,

$$
\lim _{\ell \rightarrow \infty} \frac{S_{\ell} \phi(x)}{S_{\ell} \log _{2}\left|\widehat{T}^{\prime}\right|(x)}=\frac{\frac{\partial P}{\partial q}}{\frac{\partial P}{\partial t}}=-t^{\prime}\left(q_{0}\right)=\alpha .
$$

This shows Claim (A).

Claim (B): For $\mu_{t\left(q_{0}\right), q_{0}}$ almost all $x$,

$$
\lim _{n \rightarrow \infty} \frac{\log \mu_{t\left(q_{0}\right), q_{0}}\left(I_{n}(x)\right)}{\log 2^{-n}}=t\left(q_{0}\right)+q_{0} \alpha,
$$

where $I_{n}(x)$ is the $T$-dyadic cylinder of order $n$ containing $x$.

On the one hand, by (2.6) and then by (2.8) and (2.7), one has for $\mu_{t\left(q_{0}\right), q_{0}}$ almost all $x$

$$
\lim _{\ell \rightarrow \infty} \frac{\log \mu_{t\left(q_{0}\right), q_{0}}\left(D_{\ell}(x)\right)}{\log \left|D_{\ell}(x)\right|}=t\left(q_{0}\right)+q_{0} \frac{\frac{\partial P}{\partial q}}{\frac{\partial P}{\partial t}}=t\left(q_{0}\right)+q_{0} \alpha .
$$

On the other hand, note that for any $x \in E(\alpha)$, if the binary expansion of $x$ is $x=\left[0^{n_{1}-1} 10^{n_{2}-1} 1 \ldots\right]$, then for any $\delta>0$, for $\ell$ large enough,

$$
(\alpha-\delta) \ell \leq 2^{n_{1}}+\cdots+2^{n_{\ell}}-\ell=S_{\ell} \phi(x) \leq(\alpha+\delta) \ell .
$$

Hence

which implies

$$
n_{\ell}=O(\log \ell)
$$

$$
\lim _{\ell \rightarrow \infty} \frac{\log \left|D_{\ell}(x)\right|}{\log \left|D_{\ell+1}(x)\right|}=\lim _{\ell \rightarrow \infty} \frac{n_{1}+\cdots+n_{\ell}}{n_{1}+\cdots+n_{\ell}+n_{\ell+1}}=1 .
$$


Thus

$$
\lim _{n \rightarrow \infty} \frac{\log \mu_{t\left(q_{0}\right), q_{0}}\left(I_{n}(x)\right)}{\log 2^{-n}}=\lim _{\ell \rightarrow \infty} \frac{\log \mu_{t\left(q_{0}\right), q_{0}}\left(D_{\ell}(x)\right)}{\log \left|D_{\ell}(x)\right|} .
$$

This shows Claim (B).

To conclude the desired lower bound, we apply the classical mass distribution principle (see [3, Proposition 4.2]). Since the Hausdorff dimension will not be changed if we replace the $\delta$-coverings by $T$-dyadic cylinder coverings (see [3, Section 2.4]), the lower bound of the Hausdorff dimension can be given by the mass transference principle on $T$-dyadic cylinders. By the above two claims and Egorov's theorem, for any $\eta>0$, there exists an integer $N_{0}$ such that the set

$$
\left\{x \in E_{\alpha}: \mu\left(I_{n}(x)\right) \leq\left|I_{n}(x)\right|^{t\left(q_{0}\right)+q_{0} \alpha-\eta}, n \geq N\right\}
$$

is of $\mu_{t\left(q_{0}\right), q_{0}}$ positive measure. So, it implies that

$$
\operatorname{dim}_{H} E_{\alpha} \geq t\left(q_{0}\right)+q_{0} \alpha-\eta
$$

Note that ([10, Lemma 7.5]) the function $q \mapsto t(q)$ is a decreasing convex function such that

$$
t(0)=1, \quad \lim _{q \rightarrow \infty}(t(q)+q)=0,
$$

and

$$
\lim _{q \rightarrow 0^{+}} t^{\prime}(q)=-\infty, \quad \lim _{q \rightarrow+\infty} t^{\prime}(q)=-1 .
$$

Therefore, we have proved for any $\alpha \in(1,+\infty)$

$$
\operatorname{dim}_{H}(\widetilde{E}(\alpha))=\inf _{q>0}\{t(q)+q \alpha\}
$$

which is Legendre transformation. All the properties stated in Theorem 1.1 are satisfied by the function $\alpha \mapsto \operatorname{dim}_{H}(\widetilde{E}(\alpha))$ which is the same function as $\alpha \mapsto$ $\operatorname{dim}_{H}(E(\alpha))$ by Lemma 2.1

For the end point $\alpha=1$, it suffices to note that the level set $E(1)$ is nothing but the set of numbers with frequency of the digit 1 in its binary expansion being 1. Thus the Hausdorff dimension of $E(1)$ is 0 . Hence, the Legendre transformation formula for the Hausdorff dimension of $E(\alpha)(\alpha>1)$ also holds for $\alpha=1$.

\section{FAST inCREASing BirkhofF SUM}

At first, we give two simple observations.

Lemma 3.1. Let $W$ be an integer such that $2^{t} \leq W<2^{t+1}$ for some positive integer $t$. For any $0 \leq n \leq t$, among the integers between $W$ and $W\left(1+2^{-n}\right)$, there is one $V=V(W, n)$ whose binary expansion of $V$ has at most $n+2$ digits 1 and ends with at least $t-n$ zeros.

Proof. By the assumption, we have $2^{-n} W \geq 2^{t-n}$. Thus among the $2^{-n} W$ consecutive integers from $W$ to $W\left(1+2^{-n}\right)$ there is at least one integer which is divisible by $2^{t-n}$ which means there is an integer $\ell \geq 1$ such that

$$
W \leq \ell 2^{k-n} \leq W\left(1+2^{-n}\right) .
$$

Let $V=\ell 2^{t-n}$ and note that $V$ is an integer whose binary expansion ends with at least $t-n$ zeros. Since $\ell 2^{t-n} \leq W\left(1+2^{-n}\right)<2^{t+2}$, we conclude that $\ell 2^{t-n}$ has at most $(t+2)-(t-n)=n+2$ digits 1 in its binary expansion.

In the follows, the base of the logarithm is taken to be 2 . 
Lemma 3.2. For each integer $W$, and any integer $n \leq \log W$, we can find a word $w$ with length

and for any $x \in I_{|w|}(w)$

$$
|w| \leq(n+2)(2+\log W)
$$

$$
W \leq \sum_{j=0}^{|w|-1} \varphi\left(T^{j} x\right) \leq W\left(1+2^{-n}\right) .
$$

Proof. Let $V$ be an integer given in Lemma 3.1. Then $W \leq V \leq W\left(1+2^{-n}\right)$. Moreover if we write this number $V$ in binary expansion:

$$
V=2^{t_{1}}+\cdots+2^{t_{p}}
$$

one has that $\lfloor\log W\rfloor+1 \geq t_{1}>\cdots>t_{p} \geq\lfloor\log W\rfloor-n$ and $p \leq n+2$. Consider the word

$$
w=\left(10^{t_{1}-1} 1,10^{t_{2}-1} 1, \cdots, 10^{t_{p}-1} 1\right)
$$

here the word $10^{t_{p}-1} 1$ is 1 when $t_{p}=0$. Then we can check that the length of $w$ satisfies

$$
|w|=\left(t_{1}+1\right)+\cdots+\left(t_{p}+1\right) \leq p\left(t_{1}+1\right) \leq(n+2)(2+\log W),
$$

and for any $x \in I_{|w|}(w)$,

Hence, the proof is completed.

$$
\sum_{j=0}^{|w|-1} \varphi\left(T^{j} x\right)=V
$$

We also need the following lemmas whose proofs are left for the reader.

Lemma 3.3. For any $m \geq 1$, define

$$
F_{m}=\left\{x \in(0,1]: \epsilon_{k m}(x)=1, \text { for all } k \geq 1\right\} .
$$

Then $\operatorname{dim}_{H} F_{m}=\frac{m-1}{m}$.

Lemma 3.4. [13, Lemma 4] Given a subset $\mathbb{J}$ of positive integers and an infinite sequence $\left\{a_{k}\right\}_{k=1}^{\infty}$ of 0 's and 1 's, let

$$
E\left(\mathbb{J},\left\{a_{k}\right\}_{k=1}^{\infty}\right)=\left\{x \in(0,1]: \epsilon_{k}(x)=a_{k}, \text { for all } k \in \mathbb{J}\right\} .
$$

If the density of $\mathbb{J}$ is zero, that is,

then $\operatorname{dim}_{H} E\left(\mathbb{J},\left\{a_{k}\right\}_{k=1}^{\infty}\right)=1$.

$$
\lim _{n \rightarrow \infty} \frac{1}{n} \operatorname{Card}\{k \leq n: k \in \mathbb{J}\}=0
$$

Before the proof Theorem 1.2 , we show the following lemma.

Lemma 3.5. Let $\Psi: \mathbb{N} \rightarrow \mathbb{N}$ be an increasing function such that $\Psi(n) / n \rightarrow \infty$ as $n \rightarrow \infty$. Assume that there exists a subsequence $N_{k}$ satisfying the following conditions

$$
N_{k}-N_{k-1} \rightarrow \infty, \Psi\left(N_{k}\right)-\Psi\left(N_{k-1}\right) \rightarrow \infty
$$

and

$$
\frac{\Psi\left(N_{k-1}\right)}{\Psi\left(N_{k}\right)} \rightarrow 1, \quad \frac{\log \left(\Psi\left(N_{k}\right)-\Psi\left(N_{k-1}\right)\right)}{N_{k}-N_{k-1}} \rightarrow 0,
$$

as $k \rightarrow \infty$. Then the set

$$
E_{\Psi}(1)=\left\{x \in(0,1]: \lim _{n \rightarrow \infty} \frac{1}{\Psi(n)} S_{n}(x)=1\right\}
$$

has Hausdorff dimension 1. 
Proof. Fix a large integer $m$ and write

$$
\mathcal{U}=\left\{u=\left(\epsilon_{1}, \cdots, \epsilon_{m}\right): \epsilon_{m}=1, \epsilon_{i} \in\{0,1\}, i \neq m\right\} .
$$

To avoid the abuse of notation, by the first assumption of (3.1), we assume $N_{k}-$ $N_{k-1} \gg m$ for all $k \geq 1$ by setting $N_{0}=0$ and $\Psi\left(N_{0}\right)=0$.

For each $k \geq 1$, we write

$$
W_{k}:=\Psi\left(N_{k}\right)-\Psi\left(N_{k-1}\right)
$$

and let $\left\{n_{k}\right\}$ be a sequence of integers tending to $\infty$ such that

$$
n_{k} \leq \log W_{k}, \quad n_{k} \cdot \frac{\log \left(\Psi\left(N_{k}\right)-\Psi\left(N_{k-1}\right)\right)}{N_{k}-N_{k-1}} \rightarrow 0 .
$$

By the second assumptions of (3.1) and (3.2), this sequence of $n_{k} \geq 0$ do exist.

Now for $W_{k}$ and $n_{k}$, let $w_{k}$ be the word given in Lemma 3.2. Then the length $a_{k}$ of $w_{k}$ satisfies

$$
\begin{aligned}
a_{k} & \leq\left(n_{k}+2\right)\left(2+\log W_{k}\right) \\
& =\left(n_{k}+2\right)\left(2+\log \left(\Psi\left(N_{k}\right)-\Psi\left(N_{k-1}\right)\right)\right)=o\left(N_{k}-N_{k-1}\right)
\end{aligned}
$$

and for any $x \in I_{a_{k}}\left(w_{k}\right)$,

$$
W_{k} \leq \sum_{j=0}^{a_{k}-1} \varphi\left(T^{j} x\right) \leq W_{k}\left(1+2^{-n_{k}}\right) .
$$

Define $t_{k}, \ell_{k}$ to be the integers satisfying

$$
N_{k}-N_{k-1}-a_{k}=t_{k} m+\ell_{k}
$$

for some $0 \leq \ell_{k}<m$.

Let $w_{k}(k \geq 1)$ be given as the above. We define a Cantor subset of $E_{\Psi}(1)$ as follows.

Level 1 of the Cantor subset. Define

$$
E_{1}=\left\{I_{N_{1}}\left(u_{1}, \cdots, u_{t_{1}}, 1^{\ell_{1}}, w_{1}\right): u_{i} \in \mathcal{U}, 1 \leq i \leq t_{1}\right\}
$$

For simplicity, we use $I_{N_{1}}\left(U_{1}\right)$ to denote a general cylinder in $E_{1}$.

Level 2 of the Cantor subset. This level is composed by sublevels for each cylinder $I_{N_{1}}\left(U_{1}\right) \in E_{1}$. Fix an element $I_{N_{1}}=I_{N_{1}}\left(U_{1}\right) \in E_{1}$. Define

$$
E_{2}\left(I_{N_{1}}\left(U_{1}\right)\right)=\left\{I_{N_{2}}\left(U_{1}, u_{1}, \cdots, u_{t_{2}}, 1^{\ell_{2}}, w_{2}\right): u_{i} \in \mathcal{U}, 1 \leq i \leq t_{2}\right\} .
$$

Then

$$
E_{2}=\bigcup_{I_{N_{1}} \in E_{1}} E_{2}\left(I_{N_{1}}\right) .
$$

For simplicity, we use $I_{N_{2}}\left(U_{2}\right)$ to denote a general cylinder in $E_{2}$.

From Level $k$ to $k+1$. Fix $I_{N_{k}}\left(U_{k}\right) \in E_{k}$. Define

$E_{k+1}\left(I_{N_{k}}\left(U_{k}\right)\right)=\left\{I_{N_{k+1}}\left(U_{k}, u_{1}, \cdots, u_{t_{k+1}}, 1^{\ell_{k+1}}, w_{k+1}\right): u_{i} \in \mathcal{U}, 1 \leq i \leq t_{k+1}\right\}$.

Then

$$
E_{k+1}=\bigcup_{I_{N_{k}} \in E_{k}} E_{k+1}\left(I_{N_{k}}\right) .
$$

Up to now we have constructed a sequence of nested sets $\left\{E_{k}\right\}_{k \geq 1}$. Set

$$
F=\bigcap_{k \geq 1} E_{k} .
$$


We claim that

$$
F \subset E(\Psi) \text {. }
$$

In fact, for all $x \in F$, by construction, for each $k \geq 1$,

$$
\begin{aligned}
& \sum_{n=N_{k-1}}^{N_{k}-1} \varphi\left(T^{n} x\right) \\
= & \sum_{n=N_{k-1}}^{N_{k-1}+t_{k} m-1} \varphi\left(T^{n} x\right)+\sum_{n=N_{k-1}+t_{k} m}^{N_{k-1}+t_{k} m+\ell_{k}-1} \varphi\left(T^{n} x\right)+\sum_{n=N_{k-1}+t_{k} m+\ell_{k}}^{N_{k}-1} \varphi\left(T^{n} x\right) \\
= & t_{k} O\left(2^{m}\right)+\ell_{k}+W_{k}\left(1+O\left(2^{-n_{k}}\right)\right) \\
= & O\left(\frac{\left(N_{k}-N_{k-1}\right) 2^{m}}{m}\right)+\left(\Psi\left(N_{k}\right)-\Psi\left(N_{k-1}\right)\right)\left(1+O\left(2^{-n_{k}}\right)\right) .
\end{aligned}
$$

Since $n_{k} \rightarrow \infty$ which implies $2^{-n_{k}} \rightarrow 0$ as $k \rightarrow \infty$, we have

$$
\sum_{n=0}^{N_{k}-1} \varphi\left(T^{n} x\right)=\Psi\left(N_{k}\right)(1+o(1))+O\left(\frac{N_{k} 2^{m}}{m}\right) .
$$

By the assumption $\Psi(n) / n \rightarrow \infty$ as $n \rightarrow \infty$, we then deduce

$$
\sum_{n=0}^{N_{k}-1} \varphi\left(T^{n} x\right)=\Psi\left(N_{k}\right)+o\left(\Psi\left(N_{k}\right)\right),
$$

Thus

$$
\lim _{k \rightarrow \infty} \frac{\sum_{n=0}^{N_{k}-1} \varphi\left(T^{n} x\right)}{\Psi\left(N_{k}\right)}=1 .
$$

While, for each $N_{k-1}<N \leq N_{k}$

$$
\frac{\sum_{n=0}^{N_{k-1}-1} \varphi\left(T^{n} x\right)}{\Psi\left(N_{k}\right)} \leq \frac{\sum_{n=0}^{N-1} \varphi\left(T^{n} x\right)}{\Psi(N)} \leq \frac{\sum_{n=0}^{N_{k}-1} \varphi\left(T^{n} x\right)}{\Psi\left(N_{k-1}\right)} .
$$

So by the first assumption of (3.2), we deduce from (3.5) that

$$
\lim _{n \rightarrow \infty} \frac{1}{\Psi(n)} S_{n}(x)=1 \text {. }
$$

This proves $x \in E_{\Psi}(1)$ and hence $F \subset E_{\Psi}(1)$.

In the following, we will construct a Hölder continuous function from $F$ to $F_{m}$. Recall that

$$
F_{m}=\left\{x \in(0,1]: \epsilon_{k m}(x)=1, \text { for all } k \geq 1\right\}
$$

Define

$$
\begin{aligned}
f: F & \rightarrow F_{m} \\
x & \mapsto y
\end{aligned}
$$

where $y$ is obtained by eliminating the digits $\left\{\left(\epsilon_{N_{k}-\ell_{k}-a_{k}+1}, \cdots, \epsilon_{N_{k}}\right)\right\}_{k \geq 1}$ in the binary expansion of $x$. Now we calculate the Hölder expoent of $f$.

Take two points $x_{1}, x_{2} \in F$ closed enough. Let $n$ be the smallest integer such that $\epsilon_{n}\left(x_{1}\right) \neq \epsilon_{n}\left(x_{2}\right)$ and $k$ be the integer such that $N_{k}<n \leq N_{k+1}$. Note that by the construction of $F$, the digits sequence

$$
\left\{\left(\epsilon_{N_{k}-\ell_{k}-a_{k}+1}, \cdots, \epsilon_{N_{k}}\right)\right\}_{k \geq 1} \text { and }\left\{\epsilon_{N_{k}+t m}\right\}_{1 \leq t \leq t_{k+1}}
$$

are the same for all $x \in F$. So we must have

$$
N_{k}<n<N_{k+1}-\ell_{k+1}-a_{k+1} .
$$


Since $n$ is strictly less than $N_{k+1}-\ell_{k+1}-a_{k+1}$ and $\epsilon_{N_{k}+t m}\left(x_{1}\right)=\epsilon_{N_{k}+t m}\left(x_{2}\right)=1$ for all $1 \leq t \leq t_{k+1}$, thus, at most $m$ steps after the position $n$, saying $n^{\prime}, \epsilon_{n^{\prime}}\left(x_{1}\right)=$ $\epsilon_{n^{\prime}}\left(x_{2}\right)=1$. So it follows that

$$
\left|x_{1}-x_{2}\right| \geq \frac{1}{2^{n+m}}
$$

Again by the construction and the definition of the map $f$, we have $y_{1}=f\left(x_{1}\right)$ and $y_{2}=f\left(x_{2}\right)$ have common digits up to the position $n-1-\left(\ell_{1}+a_{1}\right)-\cdots-\left(\ell_{k}+a_{k}\right)$. Thus, it follows

$$
\left|f\left(x_{1}\right)-f\left(x_{2}\right)\right| \leq \frac{1}{2^{n-1-\left(\ell_{1}+a_{1}\right)-\cdots-\left(\ell_{k}+a_{k}\right)}} .
$$

Recall that $\ell_{k}<m$ and $a_{1}+\cdots+a_{k}=o\left(N_{k}\right)$ (see (3.3)) and also that $N_{k} / k \rightarrow \infty$ as $k \rightarrow \infty$ (by (3.1)). We have

$$
1 \geq \frac{n-1-\left(\ell_{1}+a_{1}\right)-\cdots-\left(\ell_{k}+a_{k}\right)}{n+m} \geq \frac{n-1-k m-o\left(N_{k}\right)}{n+m}=1+o(1),
$$

which implies that $f$ is $(1-\eta)$-Hölder for any $\eta>0$. Thus

$$
\operatorname{dim}_{H} F \geq(1-\eta) \operatorname{dim}_{H} F_{m} \text {. }
$$

By Lemma 3.3, we then have

$$
\operatorname{dim}_{H} F \geq(1-\eta) \frac{m-1}{m}
$$

By the arbitrariness of $\eta>0$ and letting $m \rightarrow \infty$, we conclude that $\operatorname{dim}_{H} E(\Psi)=1$. This finishes the proof.

Proof of Theorem 1.2. In all the three parts of Theorem 1.2, the case of $\beta=0$ is a direct consequence of Theorem 1.1 .

(I). Assume that $\Psi$ is one of the functions $\Psi(n)=n \log n, \Psi(n)=n^{a}(a>1)$, $\Psi(n)=2^{n^{\gamma}}$ with $0<\gamma<1 / 2$.

$\left(\mathrm{I}_{1}\right) .0<\beta<\infty$. It suffices to consider the dimension of $E_{\Psi}(1)$ i.e. $\beta=1$, since for other $\beta \in(0, \infty)$, we need only replace $\Psi(n)$ by $\beta \Psi(n)$.

To show $\operatorname{dim}_{H} E_{\Psi}(1)=1$, we can apply Lemma 3.5 directly. If $\Psi(n)=n \log n$, we can choose $N_{k}=k^{2}$. For $\Psi(n)=n^{a}(a>1)$, we can also choose $N_{k}=k^{2}$. Suppose now $\Psi(n)=2^{n^{\gamma}}$ with $0<\gamma<1 / 2$. Let $\delta>0$ be small such that

$$
\frac{\gamma}{1-\gamma}+\delta \gamma<1
$$

which is possible since $\gamma<1 / 2$. Take

$$
N_{k}=\left\lfloor k^{\frac{1}{1-\gamma}+\delta}\right\rfloor \text {. }
$$

Then we have

$$
N_{k+1}-N_{k} \approx k^{\frac{\gamma}{1-\gamma}+\delta}
$$

and

$$
\begin{aligned}
\log \left(\Psi\left(N_{k+1}\right)-\Psi\left(N_{k}\right)\right) & \approx \log \left(\Psi^{\prime}\left(N_{k}\right)\left(N_{k+1}-N_{k}\right)\right) \\
& \approx N_{k}^{\gamma}+\log \left(N_{k+1}-N_{k}\right) \approx N_{k}^{\gamma} .
\end{aligned}
$$

Here we write $A \approx B$ when $A / B \rightarrow 1$. This shows the validity of (3.1). Moreover,

$$
\frac{\log \left(\Psi\left(N_{k+1}\right)-\Psi\left(N_{k}\right)\right)}{N_{k+1}-N_{k}} \approx \frac{k^{\frac{\gamma}{1-\gamma}+\gamma \delta}}{k^{\frac{\gamma}{1-\gamma}+\delta}}=k^{-\delta(1-\gamma)} \rightarrow 0(k \rightarrow \infty) .
$$

Thus the second assumption of (3.2) is satisfied. At last, for the first assumption in (3.2), by (3.7)

$$
\frac{\Psi\left(N_{k-1}\right)}{\Psi\left(N_{k}\right)}=2^{(k-1)^{\frac{\gamma}{1-\gamma}+\delta \gamma}-k^{\frac{\gamma}{1-\gamma}+\delta \gamma}} \rightarrow 1 .
$$


Hence Lemma 3.5 applies.

$\left(\mathrm{I}_{2}\right)$. If $\beta=\infty$, we may choose $\widetilde{\Psi}(n)=2^{n^{\eta}}$ for some $0<\eta<\frac{1}{2}$ such that $E_{\widetilde{\Psi}}(1) \subset E_{\Psi}(\infty)$. Then $\operatorname{dim}_{H} E_{\Psi}(\infty)=1$ follows from $\left(\mathrm{I}_{1}\right)$.

(II). Now suppose that $\Psi(n)=2^{n^{\gamma}}$ with $1 / 2 \leq \gamma<1$.

$\left(\mathrm{II}_{1}\right)$. Let $\beta \in(0, \infty)$. We will prove that $E_{\Psi}(\beta)$ is empty. On the contrary, suppose there is $x \in E_{\Psi}(\beta)$, which has binary expansion

$$
x=\left[0^{n_{1}-1} 10^{n_{2}-1} 1 \cdots 0^{n_{\ell}-1} 1 \cdots\right] .
$$

Then, by (2.1) we have

$$
\begin{aligned}
\frac{S_{n_{1}+n_{2}+\cdots+n_{\ell}}(x)}{\Psi\left(n_{1}+n_{2}+\cdots+n_{\ell}\right)} & =\frac{2^{n_{1}}+2^{n_{2}}+\cdots+2^{n_{\ell}}-\ell}{2^{\left(n_{1}+n_{2}+\cdots+n_{\ell}\right)^{\gamma}} \rightarrow \beta,} \\
\frac{S_{n_{1}+n_{2}+\cdots+n_{\ell}+1}(x)}{\Psi\left(n_{1}+n_{2}+\cdots+n_{\ell}+1\right)} & =\frac{2^{n_{1}}+2^{n_{2}}+\cdots+2^{n_{\ell}}-\ell+2^{n_{\ell+1}-1}}{2^{\left(n_{1}+n_{2}+\cdots+n_{\ell}+1\right)^{\gamma}}} \rightarrow \beta .
\end{aligned}
$$

Since

$$
\frac{2^{\left(n_{1}+n_{2}+\cdots+n_{\ell}\right)^{\gamma}}}{2^{\left(n_{1}+n_{2}+\cdots+n_{\ell}+1\right)^{\gamma}}} \rightarrow 1,
$$

by dividing the two limits of (3.11), we deduce that

$$
\frac{2^{n_{1}}+2^{n_{2}}+\cdots+2^{n_{\ell}}-\ell+2^{n_{\ell+1}-1}}{2^{n_{1}}+2^{n_{2}}+\cdots+2^{n_{\ell}}-\ell}=1+\frac{2^{n_{\ell+1}-1}}{2^{n_{1}}+2^{n_{2}}+\cdots+2^{n_{\ell}}-\ell} \rightarrow 1,
$$

which implies that

$$
\frac{S_{n_{1}+n_{2}+\cdots+n_{\ell+1}}(x)}{S_{n_{1}+n_{2}+\cdots+n_{\ell}}(x)}=1+\frac{2^{n_{\ell+1}}-1}{2^{n_{1}}+2^{n_{2}}+\cdots+2^{n_{\ell}}-\ell} \rightarrow 1 .
$$

Combining with (3.11), we get

$$
1 \leftarrow \frac{\Psi\left(n_{1}+\cdots+n_{\ell+1}\right)}{\Psi\left(n_{1}+\cdots+n_{\ell}\right)}=\frac{2^{\left(n_{1}+n_{2}+\cdots+n_{\ell}+n_{\ell+1}\right)^{\gamma}}}{2^{\left(n_{1}+n_{2}+\cdots+n_{\ell}\right)^{\gamma}}} .
$$

Thus

$$
\begin{aligned}
& \left(n_{1}+n_{2}+\cdots+n_{\ell}+n_{\ell+1}\right)^{\gamma}-\left(n_{1}+n_{2}+\cdots+n_{\ell}\right)^{\gamma} \\
& =\left(n_{1}+n_{2}+\cdots+n_{\ell}\right)^{\gamma}\left(\left(1+\frac{n_{\ell+1}}{n_{1}+n_{2}+\cdots+n_{\ell}}\right)^{\gamma}-1\right) \\
& \approx \frac{\gamma n_{\ell+1}}{\left(n_{1}+n_{2}+\cdots+n_{\ell}\right)^{1-\gamma}} \rightarrow 0 .
\end{aligned}
$$

Therefore, for any $\varepsilon>0$, there exists $k_{0} \geq 1$ such that for all $j>k_{0}$,

$$
n_{j}<\varepsilon\left(n_{1}+n_{2}+\cdots+n_{j-1}\right)^{1-\gamma} .
$$

Then for any $k_{0}<j \leq \ell$

$$
n_{j}<\varepsilon\left(n_{1}+n_{2}+\cdots+n_{\ell}\right)^{1-\gamma} .
$$

This implies

$$
\begin{aligned}
S_{n_{1}+n_{2}+\cdots+n_{\ell}}(x) & =2^{n_{1}}+2^{n_{2}}+\cdots+2^{n_{\ell}}-\ell \\
& \leq M+\ell 2^{\epsilon\left(n_{1}+n_{2}+\cdots+n_{\ell}\right)^{1-\gamma}}-\ell,
\end{aligned}
$$

with $M:=2^{n_{1}}+\cdots+2^{n_{k_{0}}}$. Thus we have

$$
\frac{S_{n_{1}+n_{2}+\cdots+n_{\ell}}(x)}{\Psi\left(n_{1}+n_{2}+\cdots+n_{\ell}\right)}<\frac{M+\ell 2^{\epsilon\left(n_{1}+n_{2}+\cdots+n_{\ell}\right)^{1-\gamma}}-\ell}{2^{\left(n_{1}+n_{2}+\cdots+n_{\ell}\right)^{\gamma}}} .
$$

By observing $n_{j} \geq 1$, we deduce that the upper bound of (3.13) converges to 0 for $1 / 2 \leq \gamma<1$, a contradiction to (3.11). Hence $E_{\Psi}(\beta)$ is an empty set. 
$\left(\mathrm{II}_{2}\right) . \quad \beta=\infty$. Fix $\delta \in(\gamma, 1)$ and take a large integer $K$ such that $2^{K \delta}>1$. Consider the set of points such that at every position $2^{k}, k>K$ in their binary expansions, they have a string of zeros of length $2^{\delta k}$, i.e.

$$
E:=\left\{x \in(0,1]: \epsilon_{2^{k}+1}=\cdots=\epsilon_{2^{k}+\left\lfloor 2^{k \delta}\right\rfloor}=0, \text { for all } k \geq K\right\} .
$$

On the one hand, $E \subset E_{\psi}(\infty)$, since for any $n \in\left(2^{k}, 2^{k+1}\right]$ for some $k \geq K$,

$$
S_{n}(x)>2^{2^{k \delta}} \geq 2^{(n / 2)^{\delta}} \gg 2^{n^{\gamma}} .
$$

On the other hand, the set $E$ has dimension 1 guaranteed by Lemma 3.4 .

(III). Suppose that $\Psi(n)=2^{n^{\gamma}}$ with $\gamma \geq 1$ and let $\beta \in(0,+\infty]$. Assume that there exists $x \in E_{\Psi}(\beta)$ for some $\beta \in(0,+\infty)$. Write the binary expansion of $x$ as (3.10). Then by (2.1),

$$
\begin{gathered}
\frac{S_{n_{1}+n_{2}+\cdots+n_{\ell}}(x)}{\Psi\left(n_{1}+n_{2}+\cdots+n_{\ell}\right)}=\frac{2^{n_{1}}+2^{n_{2}}+\cdots+2^{n_{\ell}}-\ell}{2^{\left(n_{1}+n_{2}+\cdots+n_{\ell}\right)^{\gamma}} \rightarrow \beta,} \\
\frac{S_{n_{1}+n_{2}+\cdots+n_{\ell}-1}(x)}{\Psi\left(n_{1}+n_{2}+\cdots+n_{\ell}-1\right)}=\frac{2^{n_{1}}+2^{n_{2}}+\cdots+2^{n_{\ell}}-\ell-1}{2^{\left(n_{1}+n_{2}+\cdots+n_{\ell}-1\right)^{\gamma}}} \rightarrow \beta .
\end{gathered}
$$

However

$$
\frac{2^{n_{1}}+2^{n_{2}}+\cdots+2^{n_{\ell}}-\ell}{2^{n_{1}}+2^{n_{2}}+\cdots+2^{n_{\ell}}-\ell-1} \rightarrow 1 \text { but } \frac{2^{\left(n_{1}+n_{2}+\cdots+n_{\ell}\right)^{\gamma}}}{2^{\left(n_{1}+n_{2}+\cdots+n_{\ell}-1\right)^{\gamma}}} \geq 2,
$$

which is a contradiction. Hence $E_{\Psi}(\beta)$ is empty when $\beta \in(0,+\infty)$.

When $\beta=+\infty$, by (2.1), we have

$$
\liminf _{n \rightarrow \infty} \frac{S_{n}(x)}{2^{n}} \leq 1
$$

So,

$$
\liminf _{n \rightarrow \infty} \frac{S_{n}(x)}{\Psi(n)} \leq 1 .
$$

This shows that $E_{\Psi}(\infty)$ is also empty.

\section{The potential $1 / x$}

In fact, the techniques in Section 3 can be applied to the continuous potential $g: x \mapsto 1 / x$ on $(0,1]$ which has a singularity at 0 .

Proof of Theorem 1.3. We first show that if $\Psi(n)$ is one of the following

$$
\Psi(n)=n \log n, \Psi(n)=n^{a}(a>1), \Psi(n)=2^{n^{\gamma}}(0<\gamma<1 / 2),
$$

then for any $\beta \in[0, \infty], \operatorname{dim}_{H} F_{\Psi}(\beta)=1$.

We note that if $x \in(0,1]$ has binary expansion $x=\left[0^{n} 1^{s} \ldots\right]$, then $\varphi(x)=2^{n}$ and

$$
2^{n} \leq g(x) \leq 2^{n}+2^{n-s+1}=2^{n}\left(1+2^{-s+1}\right) .
$$

In Lemma 3.2 for an integer $W$, and for any integer $n \leq \log W$, we can construct instead of the words $w=\left(10^{t_{1}-1} 1,10^{t_{2}-1} 1, \cdots, 10^{t_{p}-1} 1\right)$, the following word

$$
w=\left(10^{t_{1}-1} 1^{s+1}, 10^{t_{2}-1} 1^{s+1}, \cdots, 10^{t_{p}-1} 1^{s+1}\right) .
$$

Then the length of the word satisfies

$$
|w|=\sum_{i=1}^{p}\left(t_{i}+s+1\right) \leq p\left(t_{1}+s+1\right) \leq(n+2)(\log W+s+2) .
$$


By (4.1), for any $x \in I_{|w|}(w)$,

$$
W+s(n+2) \leq \sum_{j=0}^{|w|-1} g\left(T^{j} x\right) \leq W\left(1+2^{-n}\right) \cdot\left(1+2^{-s}\right)+2 s(n+2) .
$$

For each $k \geq 1$, we still write

$$
W_{k}:=\Psi\left(N_{k}\right)-\Psi\left(N_{k-1}\right)
$$

and let $n_{k}, s_{k}$ be a sequence of integers tending to $\infty$ such that

$$
\begin{gathered}
n_{k} \cdot \frac{\log \left(\Psi\left(N_{k}\right)-\Psi\left(N_{k-1}\right)\right)}{N_{k}-N_{k-1}} \rightarrow 0, \\
\frac{n_{k} \cdot s_{k}}{N_{k}-N_{k-1}} \rightarrow 0,
\end{gathered}
$$

and

$$
\frac{n_{k} \cdot s_{k}}{\Psi\left(N_{k}\right)-\Psi\left(N_{k-1}\right)} \rightarrow 0 .
$$

By (3.1) and (3.2), these two sequences of $n_{k} \geq 0, s_{k} \geq 0$ do exist.

Now for $W_{k}$ and $n_{k}, s_{k}$, let $w_{k}$ be the word given as above. Then by (4.3) and (4.4), the length $a_{k}$ of $w_{k}$ satisfies

$$
\begin{aligned}
a_{k} & \leq\left(n_{k}+2\right)\left(\log W_{k}+s_{k}+2\right) \\
& =\left(n_{k}+2\right)\left(\log \left(\Psi\left(N_{k}\right)-\Psi\left(N_{k-1}\right)\right)+s_{k}+2\right) \\
& =o\left(N_{k}-N_{k-1}\right)
\end{aligned}
$$

and for any $x \in I_{a_{k}}\left(w_{k}\right)$,

$$
\begin{aligned}
W_{k}+s_{k}\left(n_{k}+2\right) & \leq \sum_{j=0}^{a_{k}-1} g\left(T^{j} x\right) \\
& \leq W_{k}\left(1+2^{-n_{k}}\right) \cdot\left(1+2^{-s_{k}}\right)+2 s_{k}\left(n_{k}+2\right) .
\end{aligned}
$$

Hence by (4.5) we still have the same estimation:

$$
\sum_{n=0}^{N_{k}-1} g\left(T^{n} x\right)=\Psi\left(N_{k}\right)+o\left(\Psi\left(N_{k}\right)\right)
$$

and the rest of the proof is the same as (I) of the proof of Theorem 1.2

We can repeat the same arguments in Section 3 and show that for potential $g$, the set $F_{\Psi}(\beta)$ is empty if $\beta \in(0, \infty), \Psi(n)=2^{n^{\gamma}}(1 / 2 \leq \gamma<1)$ or $\beta \in(0, \infty], \Psi(n)=$ $2^{n^{\gamma}}(\gamma \geq 1)$.

In fact, by definition, if there exists $x \in F_{\Psi}(\beta)$, with its binary expansion

$$
x=\left[0^{n_{1}-1} 10^{n_{2}-1} 1 \cdots 0^{n_{\ell}-1} 10^{n_{\ell+1}-1} 1 \cdots\right],
$$

then

$$
\frac{S_{n_{1}+n_{2}+\cdots+n_{\ell}} g(x)}{\Psi\left(n_{1}+n_{2}+\cdots+n_{\ell}\right)} \rightarrow \beta, \quad \frac{S_{n_{1}+n_{2}+\cdots+n_{\ell}+1} g(x)}{\Psi\left(n_{1}+n_{2}+\cdots+n_{\ell}+1\right)} \rightarrow \beta
$$

Thus

$$
\frac{S_{n_{1}+n_{2}+\cdots+n_{\ell}} g(x)}{S_{n_{1}+n_{2}+\cdots+n_{\ell}+1} g(x)} \rightarrow 1 .
$$

Observing $\varphi \leq g \leq 2 \varphi$, we have

$$
\frac{2^{n_{\ell+1}}}{S_{n_{1}+n_{2}+\cdots+n_{\ell}} g(x)} \rightarrow 0,
$$


which then implies

$$
\frac{S_{n_{1}+n_{2}+\cdots+n_{\ell}} g(x)}{S_{n_{1}+n_{2}+\cdots+n_{\ell+1}} g(x)} \rightarrow 1 .
$$

By the definition of $x \in F_{\Psi}(\beta)$, we have

$$
\frac{\Psi\left(n_{1}+n_{2}+\cdots+n_{\ell+1}\right)}{\Psi\left(n_{1}+n_{2}+\cdots+n_{\ell}\right)} \rightarrow 1 .
$$

This further implies the same inequality with (3.12) and the rest of proof is the same as $\left(\mathrm{II}_{1}\right)$ and (III) by noting $\varphi \leq g \leq 2 \varphi$.

For $\Psi(n)=2^{n^{\gamma}}(1 / 2 \leq \gamma<1)$, we can also prove $\operatorname{dim}_{H} F_{\Psi}(\infty)=1$ by the same proof as $\left(\mathrm{II}_{2}\right)$.

\section{REFERENCES}

[1] J. Aaronson, An Introduction to Infinite Ergodic Theory, Mathematical Surveys and Monographs, 50. American Mathematical Society, Providence, RI, 1997. xii+284 pp.

[2] L. Barreira, B. Saussol, Variational principles and mixed multifractal spectra, Trans. Amer. Math. Soc. 353 (2001), no. 10, 3919-3944.

[3] K. J. Falconer, Fractal Geometry, Mathematical Foundations and Application, Wiley, 1990.

[4] A.-H. Fan, D.-J. Feng, and J. Wu, Recurrence, dimension and entropy, J. London Math. Soc. (2) 64 (2001), no. 1, 229-244,

[5] W. Feller, A Limit Theoerm for Random Variables with Infinite Moments, Amer. J. Math., 68 (1946), 257-262.,

[6] W. Feller, An Introduction to Probability Theory and its Applications, Vol. 1, 3rd Edition, John Wiley, 1968.

[7] A.-H. Fan, L. Liao, B.-W. Wang, J. Wu, On Khintchine exponents and Lyapunov exponents of continued fractions, Ergodic Theory Dynam. Systems 29 (2009), no. 1, 73-109.

[8] A.-H. Fan, T. Jordan, L. Liao, M. Rams, Multifractal analysis for expanding interval maps with infinitely many branches, Trans. Amer. Math. Soc. 367 (2015), 1847-1870.

[9] A.-H. Fan, L. Liao, B.-W. Wang, J. Wu, On the fast Khintchine spectrum in continued fractions, Monats. Math., 171 (2013), 329-340.

[10] P. Hanus, R. D. Mauldin and M. Urbański, Thermodynamic formalism and multifractal analysis of conformal infinite iterated function systems, Acta. Math. Hungar. 96 (2002), no. $1-2,27-98$.

[11] L. Liao, M. Rams, Subexponentially increasing sum of partial quotients in continued fraction expansions, Math. Proc. Camb. Phil. Soc., 160 (2016), 401-412.

[12] L. Liao, M. Rams, Upper and lower fast Khintchine spectra in continued fractions, Monats. Math., 180 (2016), 65-81.

[13] J. Ma, S. Wen, Z. Wen, Egoroff's theorem and maximal run length, Monatsh. Math. 151 (2007), no. 4, 287-292.

[14] E. Olivier, Analyse multifractale de fonctions continues, C. R. Acad. Sci. Paris Sér. I Math. 326 (1998), no. 10, 1171-1174.

[15] W. Philipp, Limit theorems for sums of partial quotients of continued fractions, Monats. Math., 105 (1988), 195-206.

[16] V. Sprindžuk, Metric Theory of Diophantine Approximations, V. H. Winston \& Sons, Washington, D.C., 1979.

Dong Han Kim, Department of Mathematics Education, Dongguk University-Seoul, 30 Pildong-ro 1-Gil, Jung-Gu, Seoul, 04620 Korea

E-mail address: kim2010@dongguk.edu

Lingmin liaO, LAMA, Université Paris-Est Créteil Val de Marne, 61, avenue du Général de Gaulle 94010 Créteil Cedex, France

E-mail address: lingmin.liao@u-pec.fr

Michae Rams, Institute of Mathematics, Polish Academy of Sciences, ul. Śniadeckich 8, 00-656 Warszawa, Poland

E-mail address: rams@impan.pl

Bao-Wei Wang, School of Mathematics and Statistics, Huazhong University of Science and TeChnology, 430074 Wuhan, China

E-mail address: bwei_wang@hust.edu.cn 